\title{
Lissencephaly-A Brain Flawed
}

\author{
Mathur $M^{1}$, Gupta $S^{2}$ \\ ${ }^{1}$ Dr Manoj Mathur, Associate Professor, Department of Radiodiagnosis, GMC Patiala, ${ }^{2}$ Dr Saryu Gupta, Assistant \\ Professor, Department of Radiodiagnosis, GMC Patiala, Punjab, India.
}

Address for correspondence: Dr Manoj Mathur, Email: manojnidhi66@gmail.com

\begin{abstract}
We present a case report of a twelve-day old child suffering from seizures, in which magnetic resonance imaging (MRI) established the diagnosis of Lissencephaly. Although the causes of this disease entity are manifold, MRI can reveal stigmata of antenatal maternal infections in the child. In such a scenario, cytomegalovirus (CMV) is an important offender. In this article we discuss the pathogenesis of Lissencephaly in general, along with signs of transplacental CMV infection in the child. A diagnosis of CMV induced Lissencephaly obviates the need for chromosomal analysis and other genetic studies.
\end{abstract}

Key words: Lissencephaly, Cytomegalovirus infection, Smooth brain, Cortical malformation, Agyria

\section{Introduction}

Lissencephaly refers to paucity of gyri and sulci in the cerebral cortex giving a smooth appearance to the cerebral parenchyma [1]. The condition is caused primarily by flawed neuronal migration [2]. It is therefore imperative to comprehend the dynamics of neuronal proliferation and migration in order to fathom its pathogenesis. A variety of antenatal insults ranging from genetic defects to maternal infections can produce
Lissencephaly. The child usually presents with delayed milestones, mental retardation and seizures [1]. Although, the characteristic brain appearance on crosssectional imaging allows a prompt diagnosis of the entity, Magnetic resonance imaging (MRI) can identify the stigmata of maternal infections in the child [1], thereby saving the family from the predicament of expensive and scarce genetic counselling and chromosomal analysis.

\section{Case Report}

A twelve days old male child presented with seizures, lethargy and refusal to feed. He was born at term by normal vaginal delivery, to a mother who tested positive for cytomegalovirus infection.
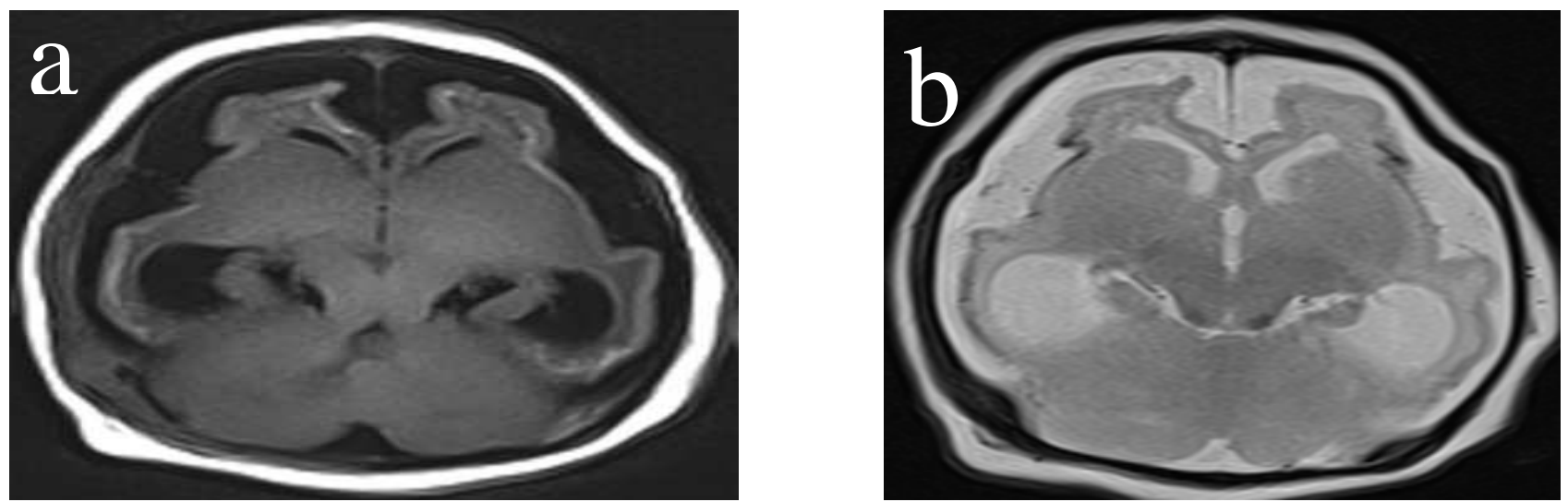

Manuscript received $2^{\text {nd }}$ May 2016

Reviewed: $12^{\text {th }}$ May 2016

Author Corrected: $24^{\text {th }}$ May 2016

Accepted for Publication $7^{\text {th }}$ June 2016 
The baby cried immediately after birth. Apgar score was normal at 8 . Birth weight was $3 \mathrm{~kg}$. Child had microcephaly. Brain MRI revealed a smooth brain with absence of gyri and sulci. Non operculized and shallow sylvian fissures resulted in a figure of 8 appearance of the cerebral hemispheres [Figure 1]. There was agenesis of the corpus callosum with dilatation of trigone and occipital horns of both lateral ventricles [Figure 2]. Brain stem was normally visualized [Figure 2].

Figure 1. Axial T1 FLAIR image (a) reveals a smooth brain with absence of gyri and sulci. The sylvian fissures are shallow and not operculized. The cortical thickness is not increased and a thin white band of calcification is seen within the cortex. Axial T2 WI (b) shows that the rim of calcification is hypointense on T2 WI.

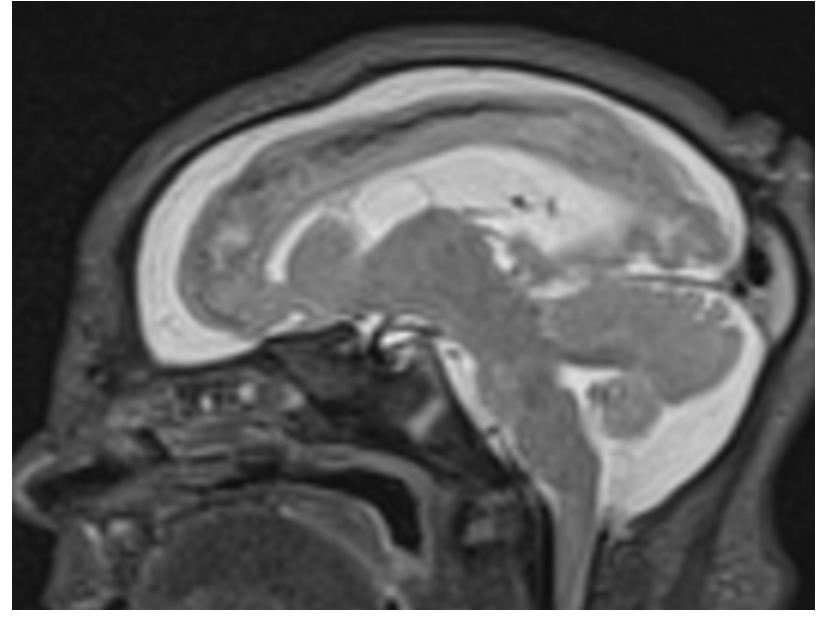

Figure 2. Sagittal T2WI shows absence of corpus callosum with colpocephaly. Brain stem is normal. Note the ventricular septations and generalised cerebral and cerebellar atrophy.

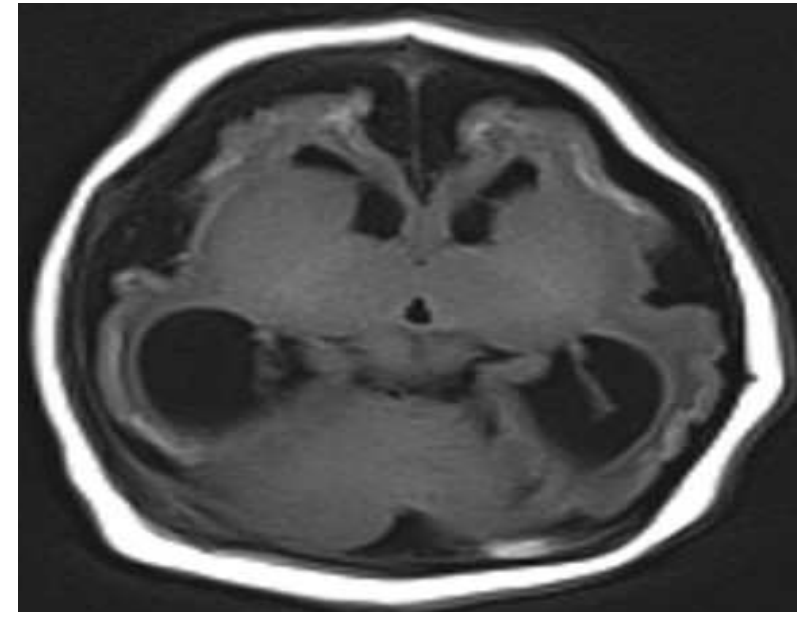

Figure-3: Axial T1 FLAIR image reveals septations in bilateral frontal horns.

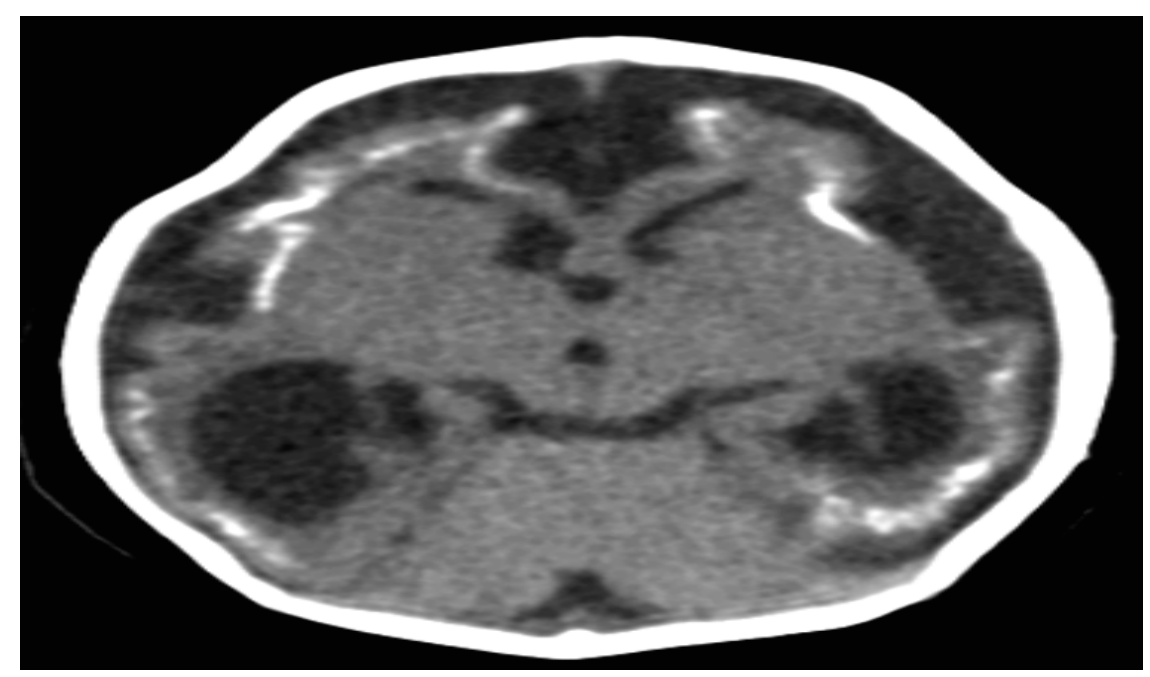

Figure-4: CT confirms the rim of calcification in the cortex. Although periventricular calcifications are characteristic, in the present case calcification was confined to the cortex.

Pertinent to note in this case was the fact, that the thickness of the cortical mantle was not increased. Ventricular septationsin bilateral frontal horns were identified [Figure 3]. This indicated the presence of ventricular adhesions. There was diffuse cerebral and cerebellar atrophy [Figure 2]. CT scan revealed a thin circumferential rim of calcification identified in bilateral cerebral cortices[Figure 4]. Therewas absence of heterotopia and periventricular calcification. Maternal ELISA was positive for CMV. PCR analysis of blood sample of the new born was positive for CMV DNA. 


\section{Discussion}

Lissencephaly refers to a smooth brain with absence or paucity of gyri and sulci. Complete absence of gyri is referred to as agyria, while paucity of gyri is pachygyria. This condition results from a disorder of neuronal migration and is associated with a thick cortex [2]. Neurons of the cerebral cortex develop during second to fourth month of gestation, in an area called the germinal matrix which lies in sub ependymal location of the lateral ventricles. From here they migrate to the cerebral cortex during third to fifth month of gestation. Initially, when the distance between the lateral ventricle and the cortical mantle is small, they do so by just elongating themselves and then pinching themselves off from the ventricular end. This process is called somal translocation $[3,4]$.

However, later in fetal life when the distance between lateral ventricle and cortical mantle increases, a more complex process is called into play. Now, the neuronal migration is facilitated by radial glial cells which span the thickness between the lateral ventricles and the cortex. This process involves the identification of neuron by the glial cell, the attachment of the neuron to the glial cell and subsequent disengagement of the two when the neuron reaches its predestined location. The disengagement is facilitated by a glycoprotein called Reelin which is produced by the first layer of the cortex. This glia-guided locomotion is used predominantly by the excitatory glutamatergic pyramidal cells of the cortex $[3,4]$.

A third route of migration involves interspersed tangential and radial movement of interneurons to reach their final designated destination. This route is followed by the GABAergic interneurons [5].

The first layer of the cortex is the first to arrive and guides the formation of subsequent layers by producing Reelin. Subsequent layers of the cortex arrive in reverse order. The seventh layer (subplate) which arrives earlyis transient and it disappears at term. Next to arrive is layer six, then five, four and so on. This implies that neurons of second layer have to traverse through layers seven, six, five, four and three to reach their rendezvous. After the arrival of the various layers of cortex in the cortical plate, the neurons interact with each other and establish synaptic connexions with other neurons. This process called cortical organization begins at the age of 22 weeks of fetal life and continues up to the age of 2 years [6].
It is therefore evident that cortical formation entails three processes- Neuronal Proliferation, Migration and Organization. These run concurrently, are mutually in weaved and show clockwork precision [6]. It can rive at any stage resulting in various disorders of corticalformation. These disordershave been classified by Barkovich [7].

Lissencephaly occurs when this organized hierarchical process gets mussed. Lissencephaly can be classified into two main types. In type I or classical Lissencephaly there is arrest of neuronal migration and a thick four layered cortex with shallow sylvian fissures is present. It is further divided into various subtypes depending up on the involvement of LIS1, relin, doublecorten and the ARX gene [7].

Type II Lissencephaly or cobblestone complex produces congenital muscular dystrophy. The condition is caused by the neurons over shooting their designated destination and thereby leading to subpial islands of neurons producing cobble stoning. Walker- Warburg syndrome and Fukuyama congenital muscular dystrophy are included in Type II Lissencephaly [8].

The most common clinical presentation in children with Lissencephaly is developmental delay and seizures $[9,10]$. Chromosomal anomalies, ischemia, maternal infections like TORCH and exogenous or endogenous toxins can result in Lissencephaly.

Congenital CMV infection is one of most common infections afflicting the fetal brain [11]. There is trans placental passage of the virus and the fetus becomes serologically reactive for CMV $[12,13]$.

The present case highlights the MR features of Lissencephaly due to transplacental fetal infection by CMV. These includethe lack in increase in cortical thickness, generalised cerebral and cerebellar atrophy, microcephaly, circumferential cortical calcification and ventricular adhesions. The lack of cortical thickening is due to less number of neurons formed in the germinal matrixcoupled with increase in programmed cell death or apoptosis in CMV induced Lissencephaly [11].

Presence of Lissencephaly in a child with prenatal infection with CMV indicates infection in early fetal life and portends a bad prognosis $[11,14,15]$. Genetic abnormalities are not detected in such cases. 


\section{Conclusion}

Maternal infection by TORCH agents are a major etiological factor in the causation of congenital anomalies in the child. Other causative factors include chromosomal anomalies, Ischemia and toxins. This case highlights the role of maternal CMV infection inducing Lissencephaly in the fetus. If antenatal maternal infection is established as the cause of Lissencephaly, genetic counselling and chromosomal analysis become redundant and the mother can hope for normal pregnancy outcome in the future.

Funding: Nil, Conflict of interest: None initiated. Permission from IRB: Yes

\section{References}

1. The CT and MR evaluation of Lissencephaly. AJNR. 1988; 9:923-927.

2. Hennekam RCM, Barth PG. Syndromic cortical dysplasias: a review. In Barth PG, ed. Disorders of neuronal migration. London MacKeith Press, 2003; 135-169.

3. Nadarajah B, Parnavelas JG. Modes of neuronal migration in the developing cerebral cortex. Nat Rev Neurosci. 2002; 3(6):423-32

4. Nadarajah B, Alifragis P, Wong ROL, Paranavelas JG. Neuronal Migration in the Developing Cerebral Cortex: observations Based on Real-time Imaging. Cereb. Cortex. 2003; 13(6): 607-611.

5. Marin O. Cellular and molecular mechanisms controlling the migration of neocortical interneurons. Eur J Neurosci. 2013; 38(1):2019-29

6. Abdel Razek AA, Kandell AY, Elsorogy LG, Elmongy A, Basett AA. Disorders of cortical formation: MR imaging features. AJNR Am J Neuroradiol. 2009 Jan; 30(1):4-11. doi: 10.3174/ajnr. A1223. Epub 2008 Aug.

7. Barkovich J, Kuzniecky RI, Jackson GD, et al. A developmental and genetic classification for malformations of cortical development. Neurology. 2005Dec 27;65(12):1873-87

8. Ghai S, Fong KW, Toi A, Chitayat D, Pantazi S, Blaser S. Prenatal US and MR imaging Findings of Lissencephaly : Review of Fetal Cerebral Sulcal Development. Radiographics. 2006; Mar-Apr 26 (2): 389-405.

9. Barkovich AJ, Raybaud CA. Neuroimaging in disorders of cortical development. Neuroimaging Clin N Am. 2004;14(2):231-54.

10. Landrieu P, Husson B, Pariente D, Lacroix C. MRIneuropathological correlations in type -1 lissencephaly. Neuroradiology. 1998 March; 40(3):173-176.

11. Fink KR, Thapa MM, Ishak GE, Pruthi S. Neuroimaging of Pediatric Central Nervous System Cytomegalovirus Infection. Radiographics. 2010; Nov 30(7): 1779-1796. doi:10.1148/rg.307105043.

12. ZuccaC, Binda S, BorgattiR, et al..Retrospective diagnosis of congenital cytomegalovirus infection and cortical maldevelopment. Neurology2003;61(5):710712. doi: http://dx.doi.org/10.1212/WNL.61.5.710

13. van der KnaapMS, VermeulenG, BarkhofF, Hart AAM, LoeberJG, WeelJFL. Pattern of white matter abnormalities at MR imaging: use of polymerase chain reaction testing of Guthrie cards to link pattern with congenital cytomegalovirus infection. Radiology2004;230(2):529-536.

14. de VriesLS, GunardiH, Barth PG, Bok LA, Verboon-MaciolekMA, GroenendaalF. The spectrum of cranial ultrasound and magnetic resonance imaging abnormalities in congenital cytomegalovirus infection. Neuropediatrics2004;35(2):113-119.

15. Barkovich AJ, Lindan CE. congenital cytomegalovirus infection of the brain: imaging analysis and embryological considerations. ajnr am $\mathrm{j}$ neuroradiol 1994; 15:703-715.

\section{How to cite this article?}

Mathur M, Gupta S. Lissencephaly- A Brain Flawed. Int J Med Res Rev 2016;4 (7):1111-1114.doi: 10.17511/ijmrr.2016.i07.07. 